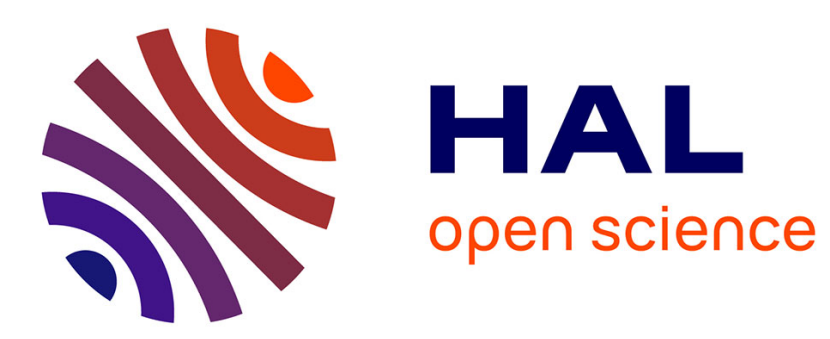

\title{
Simulation of natural convection with the Collocated Clustered Finite Volume scheme
}

\author{
O. Touazi, Eric Chénier, Robert Eymard
}

\section{To cite this version:}

O. Touazi, Eric Chénier, Robert Eymard. Simulation of natural convection with the Collocated Clustered Finite Volume scheme. Computers and Fluids, 2008, 37 (9), pp.1138-1147. 10.1016/j.compfluid.2007.09.006 . hal-00723173

\section{HAL Id: hal-00723173 \\ https://hal.science/hal-00723173}

Submitted on 3 Jun 2017

HAL is a multi-disciplinary open access archive for the deposit and dissemination of scientific research documents, whether they are published or not. The documents may come from teaching and research institutions in France or abroad, or from public or private research centers.
L'archive ouverte pluridisciplinaire HAL, est destinée au dépôt et à la diffusion de documents scientifiques de niveau recherche, publiés ou non, émanant des établissements d'enseignement et de recherche français ou étrangers, des laboratoires publics ou privés. 


\title{
Simulation of natural convection with the Collocated Clustered Finite Volume scheme
}

\author{
O. Touazi* ${ }^{*}$ E. Chénier, R. Eymard \\ Université Paris-Est, LETEM, EA 2546, 5 bd Descartes, 77454 Marne la Vallée \\ Cedex 2, France
}

\begin{abstract}
This paper presents numerical results obtained in the case of natural convection within non constant fluid density, using the Collocated Clustered Finite Volume (CCFV) scheme. The continuous equations are first given in a dimensionless form. Then we present the finite volume scheme with the principles and the spatial discretization used. Analytical tests illustrate the numerical behavior of this scheme according to the type of grid, of the pressure stabilization method and check the robustness of this scheme. Next, the results obtained on the square thermally driven cavity under large temperature differences show that the CCFV scheme accurately fits the reference results.
\end{abstract}

\section{Introduction}

Various fluid flows are successfully modeled by the Navier-Stokes equations within the Oberbeck-Boussinesq approximation: the fluid properties are assumed to be constant in the mass, momentum and energy conservation equations, except in the buoyancy term where a linear dependence of the density with respect to the temperature is taken into account. This approximation is justified when the range of relative temperature variations is small enough (the mathematical validation of this approximation is examined in $[1,2]$ ), and then leads to a simpler mathematical formulation than the complete equations. However, when the temperature gap increases significantly, the variations of the fluid properties cannot be neglected.

\footnotetext{
* Corresponding author. Tel: (33) 01609573 11; Fax: (33) 0160957294

Email addresses: Ouardia.Touazi@univ-mlv.fr, (O. Touazi),

Eric.Chenier@univ-mlv.fr, (E. Chénier), Robert.Eymard@univ-mlv.fr (R. Eymard).
} 
The Navier-Stokes and energy equations with variable fluid properties involve many non-linear terms, which have to be handled by numerical methods. Adaptations of the collocated finite volume schemes [3-5] to this case are often necessary in the engineering framework. In the Collocated Clustered Finite Volume method, the discrete unknowns are all located at the center of the cells of a unique mesh. Since the equivalent of the well-known "inf-sup" condition in the finite element setting (see [6]), or also called LBB condition (see [7]), is not satisfied by a collocated scheme, stabilization methods have to be implemented in order to avoid unrealistic pressure oscillations, sometimes called "checkerboard modes". Among the pressure stabilization techniques, let us cite the Brezzi and Pitkaranta approach [8], first introduced in the framework of the finite element method, which consists in adding in the mass conservation equation a stabilization term proportional to the laplacian of the pressure. Another common pressure stabilization technique is the "Momentum interpolation Method", presented by Rhie and Chow in [9] and usually practiced within the use of time stepping methods in finite difference or volume schemes.

We have introduced in $[4,10]$ the Collocated Clustered Finite Volume (CCFV) scheme, presenting the following characteristics:

(1) This scheme holds for a large class of grids, the only geometric requirement being that in every control volume, one can select a particular point, called the center of the control volume, such that each interface between two adjacent control volumes is orthogonal to the line joining their centers. This condition applies for rectangular or parallelepipedic meshes, triangular (2D) or tetrahedral (3D) Delaunay meshes, and Voronoï meshes.

(2) The scheme is fully coupled, and the discrete gradient operator is defined as the adjoint of the discrete divergence operator, thus ensuring the decrease of the kinetic energy for incompressible and isothermal fluid flows. The discrete non-linear mass, momentum and energy equations are simultaneously solved using an under-relaxed Newton's method, and the linear systems are solved using an iterative linear solver, namely the BiCGStab method preconditioned by an incomplete LU factorization.

(3) The pressure stabilization method is performed by selecting clusters of control volumes, i.e. small sets of control volumes, such that a stabilization term is imposed in the equation of the mass conservation, only on the edges between neighboring control volumes belonging to the same cluster. This procedure allows keeping stable discrete solutions, while the approximate solution is shown to converge to a solution of the NavierStokes equations.

We present in this paper the extension of the CCFV scheme to non constant fluid properties, thus permitting to handle the low Mach number equations for large temperature variations. Note that this extension leads to several new difficulties: 
(1) New non-linear terms have to be handled.

(2) Since the density is not constant, one has to account for cross derivative terms involved by the viscous stress tensor.

(3) The thermodynamic pressure must be computed in agreement with the temperature field.

We take advantage in this paper of the fact that the CCFV is natively a non-linear coupled scheme, the extension of which the new non-linear terms appears to be straightforward. New developments, based of recent research results on finite volume methods [11] have allowed for accounting for cross derivative terms resulting from variable density.

The outlines of this paper are the following. We give in section 2 the fluid flow equations with non constant physical properties. In section 3 are presented the numerical scheme and the main features of its practical implementation. Numerical results are then described in section 4 showing some convergence properties, the effect of the pressure stabilization method and comparisons with a benchmark, and some conclusions are drawn in section 5 .

\section{Low Mach number approximation}

The low Mach number model, suggested by Paolucci in the early 1980s [12], is used in studying very low velocity flows such as natural convection in a large temperature difference $[13,14]$. In this approach, the total pressure is the sum of a mean pressure $P^{*}(t)$ in the fluid domain $\Omega$, called the thermodynamic pressure, and fluctuations of the pressure $p^{*}\left(\vec{x}^{*}, t^{*}\right)$, supposed to be such that $\int_{\Omega} p^{*}\left(\vec{x}^{*}, t^{*}\right) \mathrm{d} v\left(\vec{x}^{*}\right)=0$ for all time $t^{*}$. From now on, the dimensional quantities are labbeled with $*$.

The dimensionless low Mach number equations for an ideal gas in an enclosure with impervious walls at the initial constant state $\left(P_{0}^{*}, T_{0}^{*}\right)$ and under the gravitational force per mass unit $-g^{*} \vec{e}_{z}$ are:

$$
\begin{gathered}
\frac{\partial}{\partial t} \rho+\vec{\nabla} \cdot(\rho \vec{v})=0 \\
\frac{\partial}{\partial t}(\rho \vec{v})+\vec{\nabla} \cdot(\rho \vec{v} \otimes \vec{v})=-\vec{\nabla} p-\frac{\operatorname{RaPr}}{\varepsilon_{T}} \rho \vec{e}_{z}+\operatorname{Pr} \vec{\nabla} \cdot \overline{\bar{\tau}} \\
\frac{\partial}{\partial t}(\rho T)+\vec{\nabla} \cdot(\rho T \vec{v})=\frac{\gamma-1}{\varepsilon_{T} \gamma} \frac{d P}{d t}+\vec{\nabla} \cdot(k \vec{\nabla} T) \\
\int_{\Omega} \rho \mathrm{d} v(\vec{x})=\int_{\Omega} \mathrm{d} v(\vec{x}), \quad \int_{\Omega} p(\vec{x}, t) \mathrm{d} v(\vec{x})=0
\end{gathered}
$$


$\overline{\bar{\tau}}$ represents the viscous stress tensor, that we assume to be given by the Stokes relation with $\overline{\bar{I}}$, the identity tensor:

$$
\overline{\bar{\tau}}=\mu\left(\vec{\nabla} \vec{v}+(\vec{\nabla} \vec{v})^{\mathrm{t}}-\frac{2}{3}(\vec{\nabla} \cdot \vec{v}) \overline{\overline{\mathrm{I}}}\right)
$$

Notice that radiative fluxes are neglected in the energy equation and the diffusive heat flux is expressed using the Fourier law $\vec{q}=-k \vec{\nabla} T$. As $\rho$ is supposed to verify the ideal gas law, we obtain:

$$
\rho=\frac{P}{1+\varepsilon_{T} T}
$$

where $\rho, \vec{v}, p, P$, and $T$ are respectively the non-dimensional density, velocity, spatially pressure fluctuations (often called dynamic pressure), thermodynamic pressure and temperature where $\rho=\rho^{*} / \rho_{0}^{*}, \vec{v}=\vec{v}^{*} / v_{0}^{*}, p=p^{*} /\left(\rho_{0}^{*} v_{0}^{* 2}\right)$, $P=P^{*} / P_{0}^{*}$ and $T=\left(T^{*}-T_{0}^{*}\right) / \Delta T^{*}$. The space variable $\vec{x}^{*}$ and velocity have been normalized by the reference height $H^{*}$, and by the ratio between the thermal diffusivity at the reference state, $a_{0}^{*}$, and $H^{*}$. The dynamic viscosity and thermal conductivity are normalized by their reference values at $\left(P_{0}^{*}, T_{0}^{*}\right)$. Three dimensionless parameters appear in these equations: the Rayleigh number $R a=\left(g^{*} H^{* 3} \varepsilon_{T}\right) /\left(a_{0}^{*} \nu_{0}^{*}\right)$, the Prandtl number $\operatorname{Pr}=\nu_{0}^{*} / a_{0}^{*}$ and the parameter $\varepsilon_{T}=\Delta T^{*} / T_{0}^{*}$.

The next section of the paper is devoted to the description of the discrete approximations of terms involving in the low Mach number model.

\section{The Collocated Clustered Finite Volume scheme}

We now intend to present the scheme for the approximation of a solution $(P, p, T, \vec{v})$ to system (1-3) with (4)-(6). Only the space discretization is presented with centered approximations. Upwind schemes and time discretization can be found in [4] and convergence proofs in [10].

In the following subsections, we first give definitions related to meshes, then we present the space discretization of system (1-3) and associated principles for this finite volume scheme. 


\subsection{Admissible meshes}

The domain $\Omega$ is completely recovered by a family $\mathcal{M}$ of unstructured convex polyhedra, called control volumes. The detailed notations for the geometric quantities involved by the discretization are illustrated in figure 1 in the $2 \mathrm{~d}$ case.

Let us introduce some notations: we denote by $\mathcal{M}$ the set of all the control volumes. For each control volume $K$, we denote by $\vec{x}_{K}$ the coordinates of its center, by $m(K)$ its area (if $d=2$ ) or its volume (if $d=3$ ), by $\mathcal{N}_{K}$ the set composed by the neighbors of $K$. We denote by $\mathcal{E}_{K \text {,int }}$ the set of the internal edges and by $\mathcal{E}_{K \text {,ext }}$, the set of the external edges. If the control volume $L$ is a neighbor of the control volume $K$, we denote by $m\left(\sigma_{K L}\right)$ the length $(d=2)$ or the area $(d=3)$ of the interface $\sigma_{K L}$ between $K$ and $L\left(\sigma_{K L} \in \mathcal{E}_{K \text {,int }}\right)$.If the control volume $K$ has an edge $\sigma$ at the boundary domain $\left(\sigma \in \mathcal{E}_{K \text {,ext }}\right)$, we denote by $m(\sigma)$ the length $(d=2)$ or the area $(d=3)$ of the interface $\sigma$ at the boundary. We denote by $\vec{x}_{\sigma}$ the orthogonal projection of $\vec{x}_{K}$ on $\sigma$, by $\vec{n}_{K, \sigma}$ the unit vector normal to $\sigma$, outward of $K$, and by $d_{K, \sigma}$, the distance between $\vec{x}_{K}$ and $\vec{x}_{\sigma}$. We then denote by $\vec{x}_{K \sigma}$ the center of gravity of the interface $\sigma$.

The main objective to elaborate this numerical scheme was its simplicity, in particular for the expression of diffusion fluxes. So the approximation of the flux normal to the edge of a control volume $\sigma_{K L}$ depends only on two adjacent cells what permits to systematically satisfy monotonic solutions for diffusive equations. To allow it, the requirements to mesh the domain are:

$$
\begin{array}{r}
\sigma_{K L} \perp \overrightarrow{x_{K} x_{L}} \\
\overrightarrow{x_{K} x_{L}} \cdot \vec{n}_{K L}>0
\end{array}
$$

From (7), we get the orthogonality property whereas relation (8) defines the order of the cell center occurence in the direction of $\vec{n}_{K L}$. These conditions are checked for rectangular or parallelepiped and dual Delaunay-Voronoï grids. Recall that a Voronoi mesh is such as the control volume $K$ is as the set of points closer to $\vec{x}_{K}$ than to any other $\vec{x}_{L}$. Therefore, the edge separating cells $K$ and $L$ belongs to the mid-plan between $\vec{x}_{K}$ and $\vec{x}_{L}$ what implies that requirements (7) and (8) are automatically checked. For triangular $(2 \mathrm{~d})$ or tetrahedral (3d) grids, the center $\vec{x}_{K}$ of the cell $K$ is the circumcenter (condition (7)); the constraint (8) is automatically verified once the Delaunay criterion is satisfied. 


\subsection{Spatial discretization of the Navier-Stokes and energy equations}

The objective in the development of this numerical scheme is that solutions of the discrete Navier-Stokes and energy equations $p_{K}, \vec{v}_{K}, T_{K}$, accurately approximate $p, \vec{v}, T$ at the point $\vec{x}_{K}$ of each control volume $K \in \mathcal{M}$. Our discrete spatial operators are also constructed so that, by multiplying the momentum and the thermal equations expressed on cell $K$ with the velocity $\vec{v}_{K}$ and the temperature $T_{K}$, and by summing over all the cells, we recover discrete approximations of the kinetic and thermal energy balances. For example, the works of the pressure forces and of the non-linear inertial forces must vanish under Boussinesq approximation.

For the sake of clarity, the discrete spatial operators are presented for enclosures with impervious walls and only for Dirichlet boundary conditions. The following notations are used in the next sections. We denote by $(A(\vec{x}))_{D}$ the discrete approximation of the continuous form $A(\vec{x})$ and by $(A(\vec{x}))_{D, K}$ the discrete approximation evaluated at the cell center $\vec{x}_{K} ; v^{(i)}$ is the i-th component of the velocity vector $\vec{v}$ and $\partial_{i}$ marks out the partial derivative into the i-th direction.

\subsubsection{Mass equation}

Let us first give the approximation of the steady mass equation integrated over the cell $K, \int_{K} \vec{\nabla} \cdot(\rho \vec{v}) \mathrm{d} v(\vec{x})$ that is:

$$
m(K)(\vec{\nabla} \cdot(\rho \vec{v}))_{D, K}=\sum_{L \in \mathcal{N}_{K}} F_{K L}
$$

where $F_{K L}$ is the approximation of the mass flux $\mathcal{F}_{K L}=\int_{\sigma_{K L}} \rho \vec{v}(\vec{x}) \cdot \vec{n}_{K L} \mathrm{~d} s(\vec{x})$ between two neighboring control volumes $K$ and $L$. Notice that no boundary contribution is introduced because of the impervious walls.

To preserve a good coupling between pressure and velocity, the approximation of the velocity crossing the edges $\sigma_{K L}$ must be second order accurate at the gravity center of the face [15]. This condition is easily fulfilled by using a linear interpolation of the velocity $\vec{v}\left(\vec{x}_{K L}\right)=\sum_{I \in \mathcal{M}} \alpha_{I} \vec{v}\left(\vec{x}_{I}\right)$. Practically, nodes $\vec{x}_{K}$ and $\vec{x}_{L}$ are involved plus, eventually, one or two other nodes close to $\vec{x}_{K L}$.

Without any loss of generality, we now suppose that only centers $\vec{x}_{K}$ and $\vec{x}_{L}$ play a part in the interpolation. This case corresponds to a mesh where the intersection point between the line $\left(\vec{x}_{K}, \vec{x}_{L}\right)$ and the edge $\sigma_{K L}$ is exactly $\vec{x}_{K L}$, the gravity center of the face $\sigma_{K L}$. Parallelepipeds in 3d [4] or rectangles and Delaunay triangles in $2 \mathrm{~d}$ satisfy these requirements. The discrete mass flux 
then writes:

$$
F_{K L}=\rho_{K L}\left(m\left(\sigma_{K L}\right)\left(a_{K L} \vec{v}_{K}+a_{L K} \vec{v}_{L}\right) \cdot \vec{n}_{K L}-\lambda_{K L} \frac{m\left(\sigma_{K L}\right)}{d_{K L}}\left(p_{L}-p_{K}\right)\right)
$$

with

$$
a_{K L}=\frac{\overrightarrow{x_{K L} x_{L}} \cdot \vec{n}_{K L}}{d_{K L}}, \quad a_{K L}+a_{L K}=1
$$

The density $\rho_{K L}$ on interface $\sigma_{K L}$ is also evaluated with a linear interpolation.

A stabilization method is necessary to prevent from the apparition of pressure oscillations in collocated schemes. We use the method presented in [4] that consists in modifying the mass equation by introducing a stabilization contribution which corresponds to the second term of the right-hand-side of equation (10), where $\lambda_{K L}$ is a stabilization parameter defined on each interface between two control volumes $K$ and $L$.

Recall briefly the principle of pressure stabilization method. The family of control volumes $\mathcal{M}$ is partitioned into disjoint subsets including groups of neighboring control volumes, with any algorithm ensuring that two control volumes belonging to the same subset are not too distant one from each other (Fig.2). An example of such an algorithm can be found in [4].

Then $\lambda_{K L}$ is chosen according to the following rule:

- $\lambda_{K L}=\lambda$ for any pair of neighboring control volumes $K$ and $L$ belonging to the same subset,

- $\lambda_{K L}=0$ otherwise.

The value $\lambda$ is a positive value, chosen large enough for preventing from the apparition of instabilities. The main advantage of gathering the cells by clusters is that the mass redistribution due to the stabilization term is limited to each cluster.

\subsubsection{Transport terms}

For $\phi \in\left\{v^{(i)}, T\right\}$, the discrete expression of the non-linear term integrated over the cell $K$ is:

$$
m(K)(\vec{\nabla} \cdot(\rho \vec{v} \phi))_{D, K}=\sum_{L \in \mathcal{N}_{K}} G_{K L}
$$


where $G_{K L}$ is the approximation of the flux $\mathcal{G}_{K L}=\int_{\sigma_{K L}}\left(\rho \vec{v}(\vec{x}) \cdot \vec{n}_{K L}\right) \phi(\vec{x}) \mathrm{d} s(\vec{x})$. To satisfy the discrete kinetic and thermal energy balances, the transport velocity must be equal to the discrete mass flux crossing each edge (Eq. (10)) and the centered approximation of $\phi$ must be used:

$$
G_{K L}=F_{K L} \frac{\phi_{K}+\phi_{L}}{2}
$$

By taking into account the mass equation for a steady flow, $\sum_{L \in \mathcal{N}_{K}} F_{K L}=0$, relation (11) also writes:

$$
m(K)(\vec{\nabla} \cdot(\rho \vec{v} \phi))_{D, K}=\sum_{L \in \mathcal{N}_{K}} F_{K L} \frac{\phi_{L}-\phi_{K}}{2}
$$

\subsubsection{Pressure}

The discrete approximation of the pressure gradient is based on its duality with the discrete velocity divergence:

$$
\sum_{K \in \mathcal{M}} m(K)\left(\vec{v}_{K} \cdot(\vec{\nabla} p)_{D, K}\right)=-\sum_{K \in \mathcal{M}}\left[\left(\sum_{L \in \mathcal{N}_{K}} m\left(\sigma_{K L}\right)\left(a_{K L} \vec{v}_{K}+a_{L K} \vec{v}_{L}\right) \vec{n}_{K L}\right) p_{K}\right]
$$

Thus we obtain

$$
m(K)(\vec{\nabla} p)_{D, K}=\sum_{L \in \mathcal{N}_{K}} m\left(\sigma_{K L}\right) a_{K L}\left(p_{L}-p_{K}\right) \vec{n}_{K L}
$$

By using the geometric property $\sum_{L \in \mathcal{N}_{K}} m\left(\sigma_{K L}\right) \vec{n}_{K L}+\sum_{\sigma \in \mathcal{E}_{K, \text { ext }}} m(\sigma) \vec{n}_{K, \sigma}=0$, another form for the pressure gradient can be established:

$$
\begin{aligned}
m(K)(\vec{\nabla} p)_{D, K}= & \sum_{L \in \mathcal{N}_{K}} m\left(\sigma_{K L}\right)\left(a_{K L} p_{L}+a_{L K} p_{K}\right) \vec{n}_{K L}+ \\
& \sum_{\sigma \in \mathcal{E}_{K, \text { ext }}} m(\sigma) p_{K} \vec{n}_{K, \sigma}
\end{aligned}
$$

Notice that relations (12) and (13) do not provide consistent approximations of the equivalent forms $\int_{K} \vec{\nabla} p \mathrm{~d} v(\vec{x})$ and $\int_{\delta K} p \vec{n} \mathrm{~d} s(\vec{x})$, except when $a_{K L}=0.5$. 


\subsubsection{Viscous diffusion}

In the low Mach number model, the viscous term integrated over the cell $K$ writes:

$$
\begin{aligned}
\int_{K} \vec{\nabla} \cdot \overline{\bar{\tau}} \mathrm{d} v(\vec{x}) & =\int_{K} \vec{\nabla} \cdot(\mu \vec{\nabla} \vec{v}) \mathrm{d} v(\vec{x})+\int_{K} \vec{\nabla} \cdot\left(\mu \vec{\nabla}^{t} \vec{v}\right) \mathrm{d} v(\vec{x})-\frac{2}{3} \int_{K} \vec{\nabla}(\mu \vec{\nabla} \cdot \vec{v}) \mathrm{d} v(\vec{x}) \\
& =\int_{K} \vec{a}(\mu, \vec{v}) \mathrm{d} v(\vec{x}) \quad+\int_{K} \vec{b}(\mu, \vec{v}) \mathrm{d} v(\vec{x}) \quad-\frac{2}{3} \int_{K} \vec{c}(\mu, \vec{v}) \mathrm{d} v(\vec{x})
\end{aligned}
$$

Let us now give the approximations of these three integrals.

- The approximation of $\vec{a}(\mu, \vec{v})$ is:

$$
m(K)(\vec{a}(\mu, \vec{v}))_{D, K}=\sum_{L \in \mathcal{N}_{K}} \vec{A}_{K L}+\sum_{\sigma \in \mathcal{E}_{K, \mathrm{ext}}} \vec{A}_{K, \sigma}
$$

where $\vec{A}_{K L}$ and $\vec{A}_{K, \sigma}$ are the approximations of $\overrightarrow{\mathcal{A}}_{K L}=\int_{\sigma_{K L}} \vec{\nabla} \vec{v}(\vec{x}) \cdot \vec{n}_{K L} \mathrm{~d} s(\vec{x})$ and $\overrightarrow{\mathcal{A}}_{K, \sigma}=\int_{\sigma} \vec{\nabla} \vec{v}(\vec{x}) \cdot \vec{n}_{K, \sigma} \mathrm{d} s(\vec{x})$. Thanks to the mesh properties (7) and (8), we simply obtain:

$$
\vec{A}_{K L}=\mu_{K L} \frac{m\left(\sigma_{K L}\right)}{d_{K L}}\left(\vec{v}_{L}-\vec{v}_{K}\right) \text { and } \vec{A}_{K, \sigma}=\mu_{\sigma} \frac{m(\sigma)}{d_{K, \sigma}}\left(\vec{v}_{\sigma}-\vec{v}_{K}\right)
$$

where $\vec{v}_{\sigma}$ is the approximation of $\vec{v}\left(\vec{x}_{\sigma}\right), \mu_{K L}$ and $\mu_{\sigma}$ are the dynamic viscosity computed on edges.

- The discrete contribution $\int_{K} \vec{b}(\mu, \vec{v}) \mathrm{d} v(\vec{x})$ is achieved by considering the weak form:

$$
\sum_{i=1}^{d} \int_{\Omega} u^{(j)} \partial_{i}\left(\mu \partial_{j} v^{(i)}\right) \mathrm{d} v(\vec{x})=-\sum_{i=1}^{d} \int_{\Omega} \partial_{i} u^{(j)} \mu \partial_{j} v^{(i)} \mathrm{d} v(\vec{x})
$$

with $u^{(j)}$ the j-th component of a vectoriel test function $\vec{u}, u^{(j)}$ being null on Dirichlet boundary conditions. By using the i-th component of the discrete strong gradient expression $\left(\partial_{i} \phi\right)_{D, K}[11]$,

$$
\begin{aligned}
\left(\partial_{i} \phi\right)_{D, K}=\frac{1}{m(K)} & \left(\sum_{L \in \mathcal{N}_{K}} \frac{m\left(\sigma_{K L}\right)}{d_{K L}}\left(\phi_{L}-\phi_{K}\right)\left(x_{K L}^{(i)}-x_{K}^{(i)}\right)+\right. \\
& \left.\sum_{\sigma \in \mathcal{E}_{\mathrm{ext}}} \frac{m(\sigma)}{d_{K, \sigma}}\left(\phi_{\sigma}-\phi_{K}\right)\left(x_{K \sigma}^{(i)}-x_{K}^{(i)}\right)\right)
\end{aligned}
$$

the discretization of the right-hand-side of (Eq. 16) writes:

$$
\left(\sum_{i=1}^{d} \int_{\Omega} \mu \partial_{i} u^{(j)} \partial_{j} v^{(i)} \mathrm{d} v(\vec{x})\right)_{D}=\sum_{i=1}^{d} \sum_{K \in \mathcal{M}} m(K) \mu_{K}\left(\partial_{i} u^{(j)}\right)_{D, K}\left(\partial_{j} v^{(i)}\right)_{D, K}
$$


The k-th component of the discrete contribution of $\int_{K} \vec{b}(\mu, \vec{v}) \mathrm{d} v(\vec{x})$ is obtained by substituting $\left(\partial_{i} u^{(j)}\right)_{D, K}$ and $\left(\partial_{j} v^{(i)}\right)_{D, K}$ by the expressions (17) for $\phi=u^{(j)}$ and $\phi=v^{(i)}$ and by choosing the test function $u_{L}^{(j)}=\delta_{K L} \delta_{j k}$ :

$$
m(K)\left(b^{(k)}(\mu, \vec{v})\right)_{D, K}=\sum_{L \in \mathcal{N}_{K}} B_{K L}^{(k)}+\sum_{\sigma \in \mathcal{E}_{K, \text { ext }}} B_{K, \sigma}^{(k)}
$$

with

$$
\begin{aligned}
& B_{K L}^{(k)}=\frac{m\left(\sigma_{K L}\right)}{d_{K L}} \sum_{i=1}^{d}\left(\mu_{K}\left(\partial_{k} v^{(i)}\right)_{D, K}\left(x_{K L}^{(i)}-x_{K}^{(i)}\right)+\mu_{L}\left(\partial_{k} v^{(i)}\right)_{D, L}\left(x_{L}^{(i)}-x_{K L}^{(i)}\right)\right) \\
& B_{K, \sigma}^{(k)}=\frac{m(\sigma)}{d_{K, \sigma}} \sum_{i=1}^{d}\left(\mu_{K}\left(\partial_{k} v^{(i)}\right)_{D, K}\left(x_{K \sigma}^{(i)}-x_{K}^{(i)}\right)+\mu_{L}\left(\partial_{k} v^{(i)}\right)_{D, L}\left(x_{L}^{(i)}-x_{L \sigma}^{(i)}\right)\right)
\end{aligned}
$$

- The k-th component of discrete contribution of $\int_{K} \vec{c}(\mu, \vec{v}) \mathrm{d} v(\vec{x})$ writes:

$$
m(K)\left(c^{(k)}(\mu, \vec{v})\right)_{D, K}=\sum_{L \in \mathcal{N}_{K}} C_{K L}^{(k)}+\sum_{\sigma \in \mathcal{E}_{K, \text { ext }}} C_{K, \sigma}^{(k)}
$$

with

$$
\begin{aligned}
& C_{K L}^{(k)}=\frac{m\left(\sigma_{K L}\right)}{d_{K L}} \sum_{i=1}^{d} \mu_{K}\left(\partial_{i} v^{(i)}\right)_{D, K}\left(x_{K L}^{(k)}-x_{K}^{(k)}\right)+\frac{m\left(\sigma_{K L}\right)}{d_{K L}} \sum_{i=1}^{d} \mu_{L}\left(\partial_{i} v^{(i)}\right)_{D, L}\left(x_{L}^{(k)}-x_{K L}^{(k)}\right) \\
& C_{K, \sigma}^{(k)}=\frac{m(\sigma)}{d_{K, \sigma}} \sum_{i=1}^{d} \mu_{K}\left(\partial_{i} v^{(i)}\right)_{D, K}\left(x_{K \sigma}^{(k)}-x_{K}^{(k)}\right)+\frac{m(\sigma)}{d_{K, \sigma}} \sum_{i=1}^{d} \mu_{L}\left(\partial_{i} v^{(i)}\right)_{D, L}\left(x_{L}^{(k)}-x_{L \sigma}^{(k)}\right)
\end{aligned}
$$

Note that the use of the strong gradient (17) in $\int_{K} \vec{a}(\mu, \vec{v}) \mathrm{d} v(\vec{x})$ may give rise to numerical instabilities. Therefore, to prevent from these oscillations, relation (14) must absolutly be applied instead.

\subsubsection{Thermal diffusion}

In the energy equation, the discrete contribution $(h(k, T))_{D, K}$ provided by the approximation of the diffusion term $\left.\int_{K} h(k, T) \mathrm{d} v(\vec{x})=\int_{K} \vec{\nabla} \cdot(k \vec{\nabla} T)\right) \mathrm{d} v(\vec{x})$ is similar to relations (14) with (15):

$$
m(K)(h(k, T))_{D, K}=\sum_{L \in \mathcal{N}_{K}} H_{K L}+\sum_{\sigma \in \mathcal{E}_{K, \text { ext }}} H_{K, \sigma}
$$

The approximations $H_{K L}$ and $H_{K, \sigma}$ of the conducted heat fluxes on $\sigma_{K L}$, $\mathcal{H}_{K L}=\int_{\sigma_{K L}} k \vec{\nabla} T(\vec{x}) \cdot \vec{n}_{K L} \mathrm{~d} s(\vec{x})$, and on $\sigma, \mathcal{H}_{K, \sigma}=\int_{\sigma} k \vec{\nabla} T(\vec{x}) \cdot \vec{n}_{K, \sigma} \mathrm{d} s(\vec{x})$, write: 


$$
H_{K L}=k_{K L} \frac{m\left(\sigma_{K L}\right)}{d_{K L}}\left(T_{L}-T_{K}\right) \text { and } H_{K, \sigma}=k_{\sigma} \frac{m(\sigma)}{d_{K, \sigma}}\left(T_{\sigma}-T_{K}\right)
$$

where $T_{\sigma}$ is the approximation of $T\left(\vec{x}_{\sigma}\right), k_{K L}$ and $k_{\sigma}$ are thermal conductivities on $\sigma_{K L}$ and $\sigma$.

\subsection{Discrete equations and solvers}

We get a system of non-linear equations, which depends on the unknowns of the control volume $K$ itself, on the unknowns of the neighbors $L \in \mathcal{N}_{K}$ of $K$ or on boundary $\sigma \in \mathcal{E}_{K \text {,ext }}$, and also, because of $(\vec{b}(\mu, \vec{v}))_{D}$ and $(\vec{c}(\mu, \vec{v}))_{D}$ exist, on unknowns of the neighbors $M \in \mathcal{N}_{L}$ of $L$. Note that under the Boussinesq approximation, the mass equation $\vec{\nabla} \cdot \vec{v}=0$ implies $\vec{c}(\mu, \vec{v})=\overrightarrow{0}$ and if furthermore the viscosity is constant, $\vec{b}(\mu, \vec{v})=\overrightarrow{0}$. In that case, the equation on cell $K$ only depends on its neighbors $L \in \mathcal{N}_{K}$ or on boundary $\sigma \in \mathcal{E}_{K \text {,ext }}$.

In our implementation, the non-linear system is solved using an under-relaxed Newton method. The resulting linear equations are then solved by an iterative linear solver using the BiCGStab method preconditioned by an incomplete LU factorization, with variable fill-in levels (a detailed description can be found in [4]). The thermodynamic pressure $P$ is then updated at the end of each Newton iteration, solving the discrete form of the total mass conservation equation given in (4).

\section{Natural convection results}

The computational domain is meshed either by rectangular cells or by Delaunay triangles thanks to a free software developed by Saltel and Hecht [16].

\subsection{Analytical tests}

We consider the stationary fluid flow and heat transfer in a square cavity with a low Mach number model. The dimensionless velocity, pressures, density and temperature fields are solutions of equations (1-6) where the time derivatives are zero. No-slip boundary conditions are applied on the isothermal walls.

To study the accuracy of the numerical method in the non-Boussinesq regime, forcing terms are added in the momentum and energy equations in order to converge towards the prescribed solution: 


$$
\begin{aligned}
P & =1 \\
T & =\sin ^{2}\left(\pi x^{(1)}\right) \sin ^{2}\left(\pi x^{(2)}\right) \\
\rho & =\frac{P}{1+\varepsilon_{T} T} \\
p & =\sin ^{2}\left(\pi x^{(1)}\right) \sin ^{2}\left(\pi x^{(2)}\right) \\
v^{(1)} & =\frac{\partial \Psi}{\partial x^{(2)}}=2 \pi \cos \left(\pi x^{(2)}\right) \sin \left(\pi x^{(2)}\right) \sin ^{2}\left(\pi x^{(1)}\right) \\
v^{(2)} & =-\frac{\partial \Psi}{\partial x^{(1)}}=-2 \pi \cos \left(\pi x^{(1)}\right) \sin \left(\pi x^{(1)}\right) \sin ^{2}\left(\pi x^{(2)}\right)
\end{aligned}
$$

The velocity and the density (i.e. the temperature) are chosen so that $\vec{\nabla} \cdot(\rho \vec{v})=$ 0 . Sutherland laws are used to evaluate the dimensionless dynamical viscosity as a function of the temperature:

$$
\mu=\left(\varepsilon_{T} T+1\right)^{3 / 2}\left(\frac{1+S^{*} / T_{0}^{*}}{1+\varepsilon_{T} T+S^{*} / T_{0}^{*}}\right)
$$

where $S^{*}=110.5 \mathrm{~K}$ is the Sutherland constant. The variation laws of the thermal conductivity $k$ and dynamical viscosity $\mu$, as a function of the temperature, are supposed identical, and computations are carried out with $R a=1$ and $\operatorname{Pr}=1$. The deviation from the analytical solution is measured by the discrete $L_{2}$-norm:

$$
\|e(\phi)\|_{2}^{2}=\sum_{K \in \mathcal{M}} m(K)\left(\phi_{K}-\phi\left(\vec{x}_{K}\right)\right)^{2}, \phi \in\left\{v^{(i)}, T, p, \rho\right\}
$$

\subsubsection{Spatial accuracy}

Figures 3a-3d show the $L_{2}$-norm of errors between the analytical and numerical solutions as a function of mean mesh size $h=1 / \sqrt{N}, N$ being the number of triangles, and with three reduced temperature differences, $\varepsilon_{T} \in\{0,1,2\}$. Remark that the Boussinesq approximation is recovered when $\varepsilon_{T}=0$. For all $\varepsilon_{T}$, the velocity, temperature and density are second-order accurate whereas the pressure is, like most of collocated schemes, first-order accurate only.

\subsubsection{Comparison of pressure stabilization methods}

The simulations are performed for the Boussinesq model, namely with $\varepsilon_{T}=$ 0 , and for Delaunay triangular meshes. Our pressure stabilization method, described in section 3.2.1, is compared with two other methods.

- The Brezzi and Pitkaranta method (BP) [8] consists in introducing a stabilization contribution $\lambda h^{\alpha}(\Delta p)_{D}$ in the mass equation, where $\left.\alpha \in\right] 0,2[$, 
$h$ is the characteristic mesh size, $\alpha \in \Re$ is a positive small value and $\Delta_{D} p \equiv a_{D}(1, p)$ is the discrete laplacian operator applied on pressure and defined by (14):

$$
(\vec{\nabla} \cdot \vec{v})_{D}=\lambda h^{\alpha}(\Delta p)_{D}
$$

- The Rhie and Chow interpolation method (RC) [9] can also be viewed as a penalization technique of the mass equation:

$$
(\vec{\nabla} \cdot \vec{v})_{D}=\lambda\left((\Delta p)_{D}-\left(\vec{\nabla} \cdot(\vec{\nabla} p)_{D}\right)_{D}\right)
$$

where the second term of the right-hand-side is constructed with the strong gradient (17).

In figures 4a-4b are drawn the velocity and pressure errors as a function of $\lambda h^{\alpha}$, for three pressure stabilization techniques and for a mesh size $h=0.019$. The accuracy of the all solutions decreases if the coefficient $\lambda h^{\alpha}$ is too small: the pressure stabilization is insufficient and oscillations appear. For large coefficients, the errors for the velocity and pressure increase except when the stabilization is based on clusters. Our clusterization technique is very few sensitive to $\lambda h^{\alpha}$ but the (RC)-method seems to provide more accurate pressure solutions for a wide gap of the coefficient.

Figures 5a-5d illustrate the convergence properties of these methods as a function of $h$ and for two coefficients, $\lambda \in\{1,0.0001\}$. For $\lambda=1$, the slopes of the curve for the (BP)-method become worse, less than 2 and 1 for the velocity and pressure respectively. No significant difference distinguishes the convergence behavior of the cluster and (RC)- methods. The advantage of the cluster stabilization method with respect to the Rhie and Chow technique stems from the stability property of the resulting scheme. While the clusters and (BP)- methods introduce stable contributions in the discrete scheme and thus provide a $L_{2}$ stability for unsteady isothermal solutions $[4,10,17,18]$, no such property can easily be proved for the (RC)-method because of the negative contribution $-\left(\vec{\nabla} \cdot(\vec{\nabla} p)_{D}\right)_{D}$ in the mass equation.

\subsection{Benchmark comparisons}

We consider the problem handled in the benchmark [19], stated in the above dimensionless form, which concerns the natural convection flow in a differentially heated square cavity. The vertical walls are isothermal at the uniform temperatures $T(0, y)=T_{h}=1 / 2$ and $T(1, y)=T_{c}=-1 / 2$ with $0 \leq y \leq 1$, and the bottom and top boundaries are adiabatic. The ratio $\varepsilon_{T}$ is given and equal to 1.2, and therefore the Boussinesq approximation no longer holds. The other dimensionless numbers are $\operatorname{Pr}=0.71, \gamma=1.4, \mathrm{Ra}=10^{6}$ or $\mathrm{Ra}=10^{7}$. As in [19], we use the Sutherland law (Eq. 20) for the viscosity and assume 
$\mu(T) / k(T)$ constant, which leads to a similar law for the thermal conductivity. We recall the definition of the local, $\mathrm{Nu}(\vec{x})$, and average, $\widehat{\mathrm{Nu}}_{x}$, Nusselt numbers:

$$
\mathrm{Nu}(\vec{x})=k(T(\vec{x})) \vec{\nabla} T(\vec{x}) \cdot \vec{n}_{\partial \Omega}(\vec{x}) \text { and } \widehat{\mathrm{Nu}}_{x}=\int_{0}^{1} \mathrm{Nu}(\vec{x}) \mathrm{dy},
$$

The computations were performed either on a non-uniform distribution of Delaunay triangles or on different orthogonal grids where the sides of the rectangular control volumes follow a sinusoidal law in each direction to accurately represent the boundary layers. The stabilization parameter is equal to $\lambda=10^{-6}$ and the iLU preconditioning is based on a three levels of fill-in.

\subsubsection{Rayleigh number $10^{6}$}

In Table 1 are indicated the relative errors, with respect to solutions obtained with our scheme with a $304 \times 304$ rectangular grid, for the results computed on Delaunay triangles and drawn from [19]. Relative differences of benchmark solutions being smaller than 1\%, our reference result is in good agreement with the literature. Bold underlines values smaller than $0.005 \%$ and show the good agreement with Vierendeels's results. The accuracy of results obtained with Delaunay triangular cells are good, except for the Nusselt number along the cold wall, $\max _{y}(\mathrm{Nu}(1 ; y))$ and $\min _{y}(\mathrm{Nu}(1 ; y))$. For non-Boussinesq flow model, the boundary layer along the cold wall is thicker than it is along the hot wall. The mesh size used being identical along the two vertical walls, it was therefore obvious that the accuracy of the heat transfer will be worst on the cold surface. The minima of the Nusselt number value are also larger than their corresponding maxima. The explanation is due to the fact that the coordinates, where $\min _{y}(\mathrm{Nu}(0 ; y))$ and $\min _{y}(\mathrm{Nu}(1 ; y))$ occur, are very close to the horizontal walls where a finer mesh should be desirable too.

The effect of the mesh size was also studied but it is not presented in this paper. Quadratic convergence has successfully been checked for the set of results except for minimal values of the Nusselt number when triangular meshes were considered.

\subsubsection{Rayleigh number $R a=10^{7}$}

For $\mathrm{Ra}=10^{7}$, only results obtained with rectangular grid are shown, the computational cost to solve the Navier-Stokes and energy equations on a fine enough triangular grid was judged too expensive. As shown in Table 2, our results are in excellent agreement with benchmark solutions [19]. Relative differences less than $0.005 \%$ are anew exhibited in bold. As for $\mathrm{Ra}=10^{6}$, the 
second order accuracy of the scheme was successfully checked for the set of results.

\section{Conclusion}

Extention of the Collocated Clustered Finite Volume scheme to the low Mach number model was presented in detail. Convergence property of the new scheme was checked by considering an analytical solution. Effect of the pressure stabilization method was studied with respect to other stabilization techniques. Applications to natural convection problems with large temperature differences have led to results which are in good agreement with the literature. 


\section{References}

1. Gray D, Giorgini A. The validity of the Boussinesq approximation for liquids and gases. Int J Heat and Mass Transfer 1976;19:545-551

2. Mihaldjan JM. A rigourous exposition of the Boussinesq approximations applicable to a thin layer of fluid. Astrophys J 1962;136(3):1126-1133

3. Archambeau F, Mehitoua N, Sakiz M. Code Saturne: A Finite Volume Code for Turbulent flows. Int J Finite Volumes 2004 "http://averoes.math.univparis13.fr/IJFV/

4. Chénier E, Eymard R, Touazi O. Numerical results using a colocated finite volume scheme on unstructured grids for incompressible fluid flows. Numer Heat Transfer, part B 2006;49:259-276

5. Mathur SR, Murthy JY. Pressure boundary conditions for incompressible flow using unstructured meshes. Numer heat transfer 1997;32:283-298

6. Pironneau O. Finite element methods for fluids. John Wiley \& Sons; 1989

7. Brezzi F. On the existence uniqueness and approximation of saddlepoint problems arising from Lagragian multipliers. RAIRO Anal Numer $1974 ; 8: 129-151$

8. Brezzi F, Pitkaranta J. On the stabilization of finite element approximations of the Stokes equations. Notes Numer Fluid Mech, Vieweg, Braunschweig $1984 ; 10$

9. Rhie CM, Chow WL. Numerical study of the turbulent flow past an airfoil with trailing edge separation. AIAA J 1983;21:1525-1532

10. Eymard R, Herbin R, Latché JC, Piar B. On colocated clustered finite volume schemes for incompressible flow problems. submitted http://hal.archives-ouvertes.fr/hal-00136125

11. Eymard R, Gallouët T, Herbin R. A cell-centred finite volume approximation for anisotropic diffusion operators on unstructured meshes in any space dimension. IMA J Numer Anal 2006;26(2):326-353 
12. Paolucci S. Filtering of sound from the Navier-Stokes equations. Sandia National Lab Report, 1982:82-8257

13. Paolucci S, Chenoweth DR. Transition to chaos in a differentially heated vertical cavity. J Fluid Mech 1989;201:379-410

14. Le Quéré P, Masson R, Perrot P. A Chebyshev Collocation Algorithm for 2D Non-Boussinesq Convection. J Comput Phys 1992;103:320-335

15. Touazi O, Etude numérique d'un schéma de volumes finis sur des maillages non structurés pour des écoulements de fluide visqueux à densité constante ou faiblement variable. Thesis of University of Marne-la-Vallée. France 2006

16. Saltel E, Hecht F. EMC ${ }^{2}$ Un logiciel d'édition de maillages et de contours bidimensionnels. INRIA 1995;118

17. Eymard R, Herbin R, Latché JC. On a stabilized colocated Finite Volume scheme for the Stokes problem. M2AN 2006;40(3):501-527

18. Eymard R, Herbin R, Latché JC. Convergence Analysis of a Colocated Finite Volume Scheme for the Incompressible Navier-Stokes Equations on General 2D or 3D Meshes. SIAM J Numer Anal 2007;45(1):1-36

19. Paillère H, Le Quéré P, Weisman C, Vierendeels J, Dick E, Braack M, Dabbene F, Beccantini A, Studer E, Kloczko T, Corre C, Heuveline V, Darbandi M, Hosseinizadeh SF. Modelling of natural convection flows with large temperature differences: A benchmark problem for low Mach number solvers ESAIM, Math Model Numer Anal 2005;39(3):617-621 


\section{List of Figures}

1 Two neighboring control volumes $K$ and $L$. One assumes that for all control volume $K$ there exists a point denoted by $\vec{x}_{K}$ in $K$ such that, for any neighbor $L$ of $K$, the straight line $\left(\vec{x}_{K}, \vec{x}_{L}\right)$ is orthogonal to the common edge $\sigma_{K L}$. Then the length between $\vec{x}_{K}$ and $\vec{x}_{L}$ is denoted by $d_{K L}$, and the unit vector, normal to $\sigma_{K L}$ and outward to $K$, is denoted by $\vec{n}_{K L}$. We then denote the center of gravity of the interface $\sigma_{K L}$ by $\vec{x}_{K L}$. Similar notations are used when an edge $\sigma$ belongs to $\delta \Omega$, the boundary of the domain $\Omega$.

2 Delaunay grid and associated clusters

3 Spatial accuracy for velocity, pressure, temperature and density

4 Effect of the stabilization parameter $\lambda h^{\alpha}$ on the $L_{2}$-norm of the velocity and pressure, $\alpha=0$ for the cluster method and the Rhie and Chow technique, (RC), whereas $\alpha=1$ for the Brezzi and Pitkaranta method, (BP).

5 Velocity and pressure errors as a function of $h$ and $\lambda$ for the cluster, Rhie and Chow (RC) and Brezzi and Pitkaranta (BP) methods. 


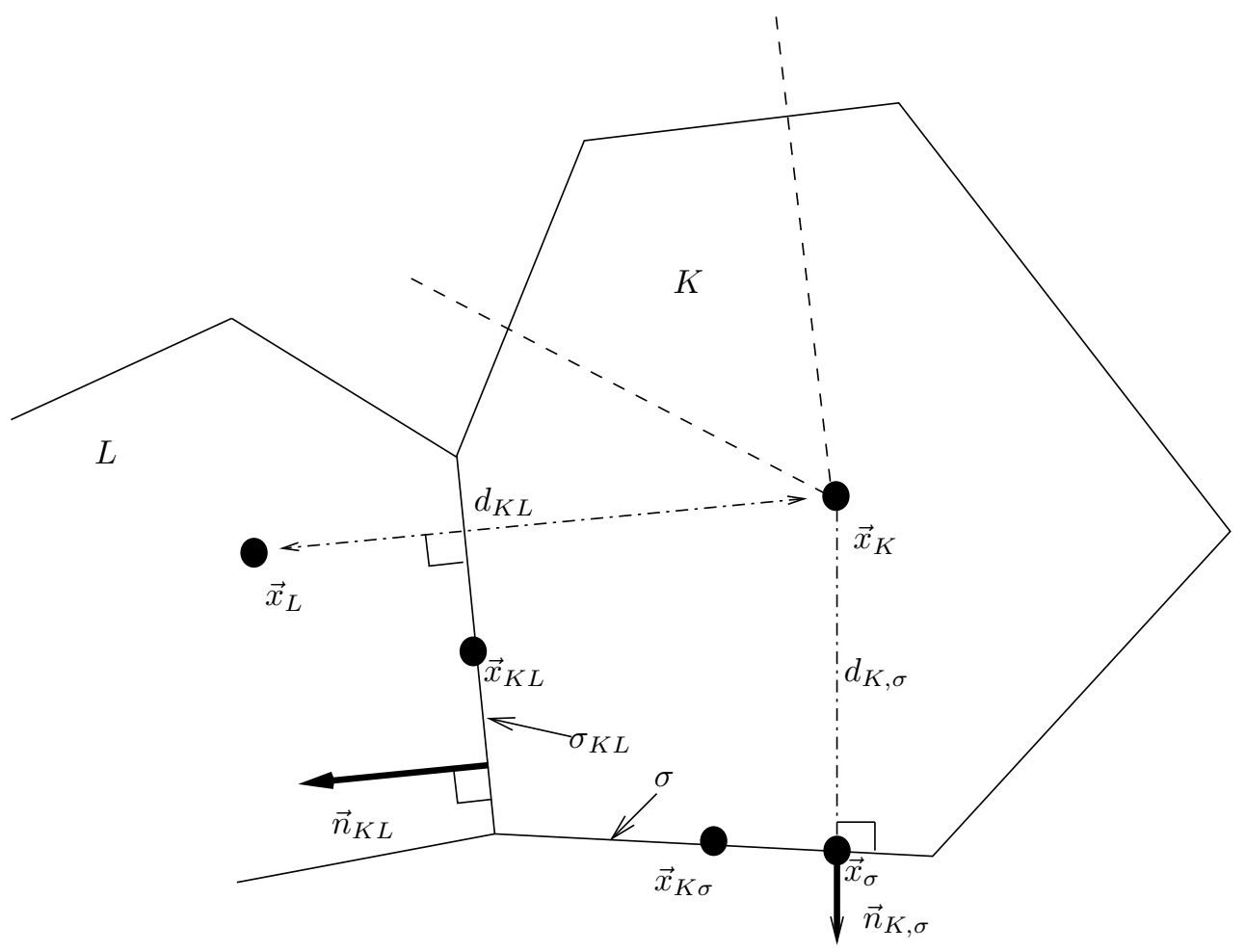

Fig. 1. Two neighboring control volumes $K$ and $L$. One assumes that for all control volume $K$ there exists a point denoted by $\vec{x}_{K}$ in $K$ such that, for any neighbor $L$ of $K$, the straight line $\left(\vec{x}_{K}, \vec{x}_{L}\right)$ is orthogonal to the common edge $\sigma_{K L}$. Then the length between $\vec{x}_{K}$ and $\vec{x}_{L}$ is denoted by $d_{K L}$, and the unit vector, normal to $\sigma_{K L}$ and outward to $K$, is denoted by $\vec{n}_{K L}$. We then denote the center of gravity of the interface $\sigma_{K L}$ by $\vec{x}_{K L}$. Similar notations are used when an edge $\sigma$ belongs to $\delta \Omega$, the boundary of the domain $\Omega$. 


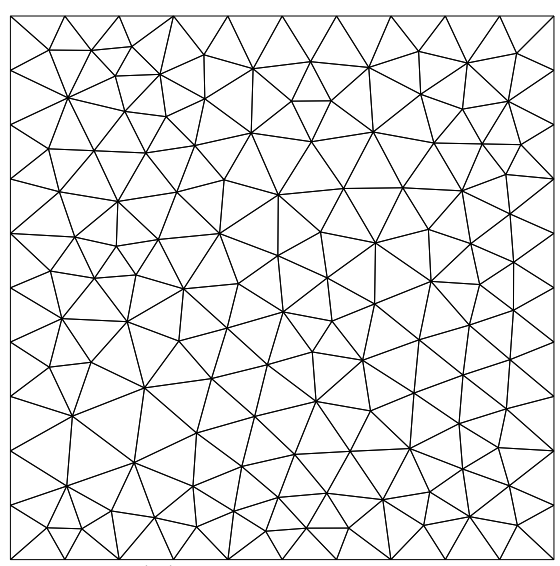

(a) Delaunay grid

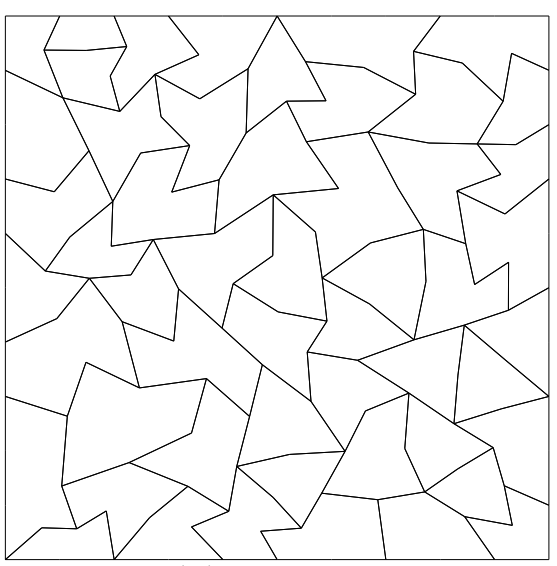

(b) Clusters

Fig. 2. Delaunay grid and associated clusters 


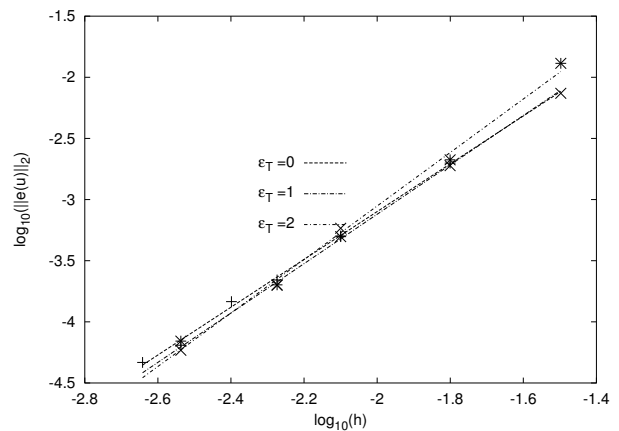

(a) $\|e(u)\|_{2}$

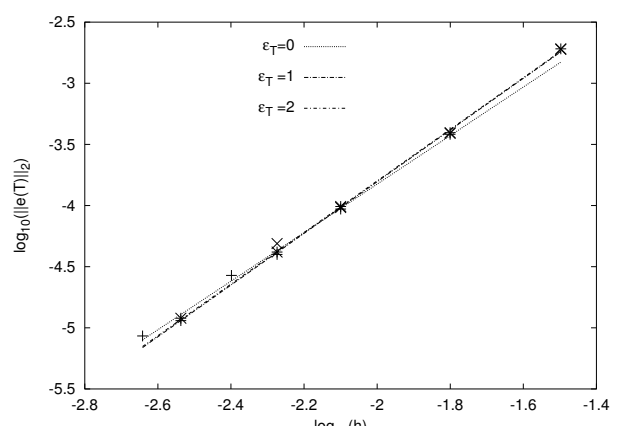

(c) $\|e(T)\|_{2}$

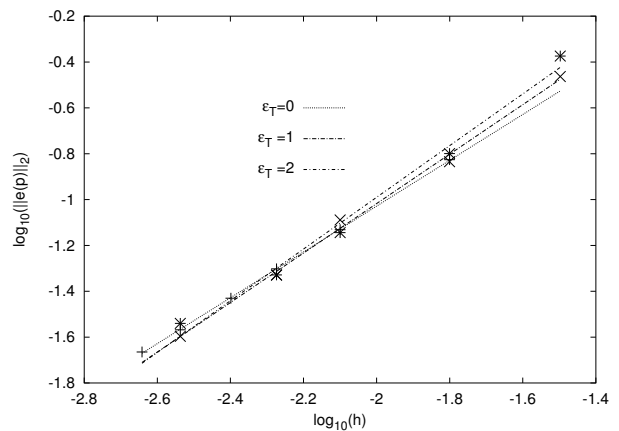

(b) $\|e(p)\|_{2}$

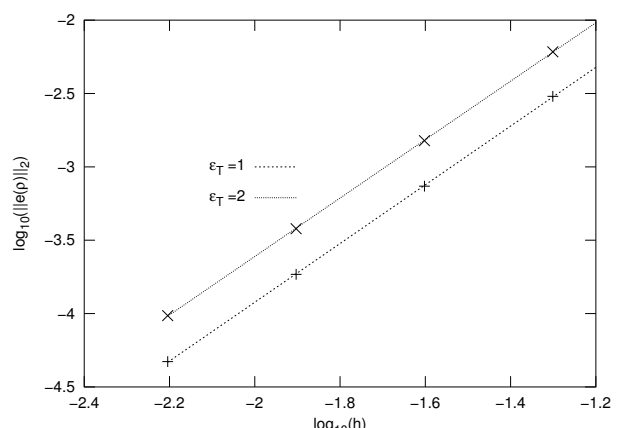

(d) $\|e(\rho)\|_{2}$

Fig. 3. Spatial accuracy for velocity, pressure, temperature and density 


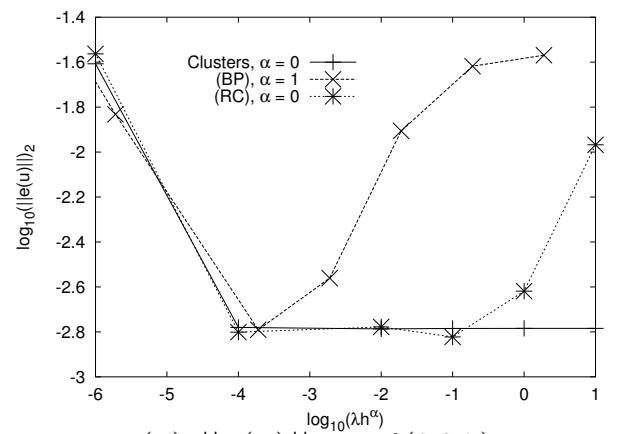

(a) $\|e(u)\|_{2}=f\left(\lambda h^{\alpha}\right)$

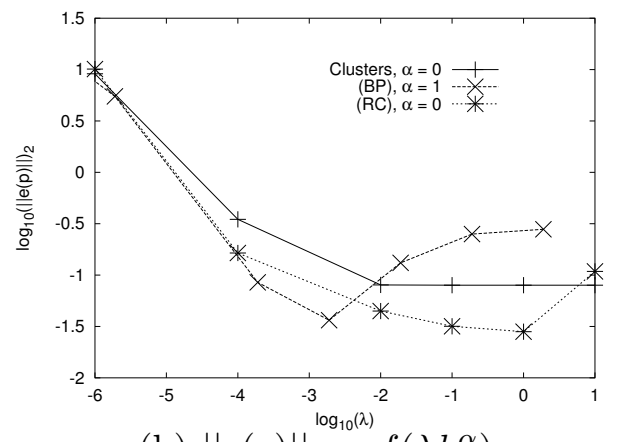

(b) $\|e(p)\|_{2}=f\left(\lambda h^{\alpha}\right)$

Fig. 4. Effect of the stabilization parameter $\lambda h^{\alpha}$ on the $L_{2}$-norm of the velocity and pressure, $\alpha=0$ for the cluster method and the Rhie and Chow technique, (RC), whereas $\alpha=1$ for the Brezzi and Pitkaranta method, (BP). 


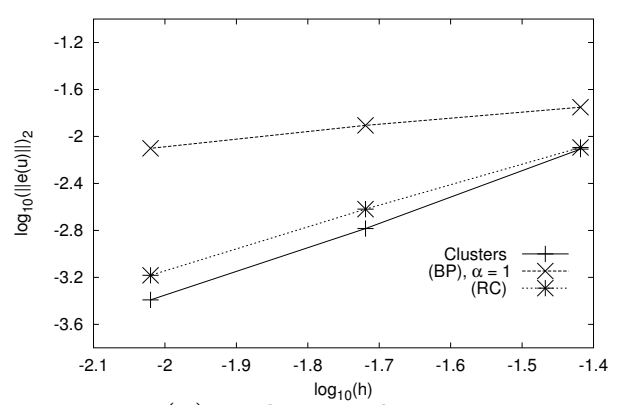

(a) Velocity, $\lambda=1$

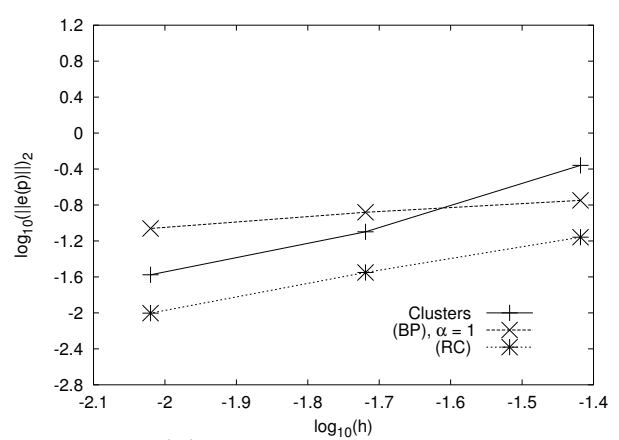

(c) Pressure, $\lambda=1$

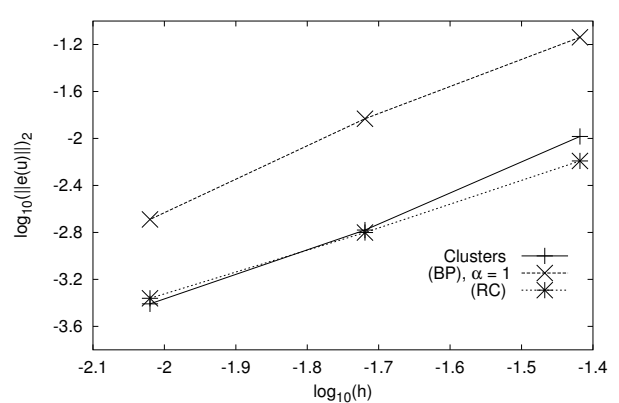

(b) Velocity, $\lambda=0.0001$

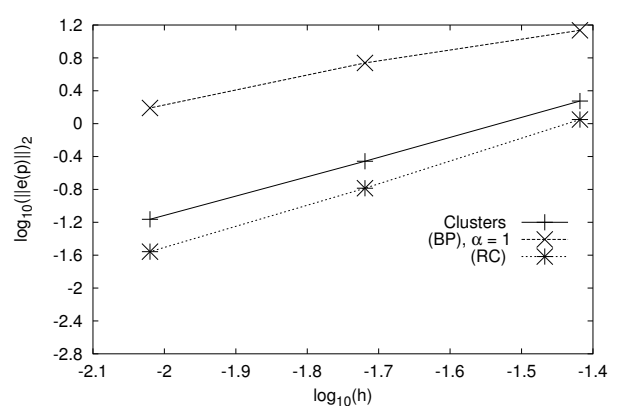

(d) Pressure, $\lambda=0.0001$

Fig. 5. Velocity and pressure errors as a function of $h$ and $\lambda$ for the cluster, Rhie and Chow (RC) and Brezzi and Pitkaranta (BP) methods. 


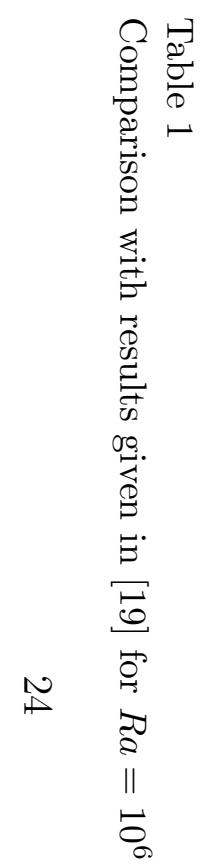

\begin{tabular}{|c|c|c|c|c|c|c|c|}
\hline & Vierendeels & Dabbene & Beccantini & Kloczko & Heuveline & $\begin{array}{c}\text { Our results } \\
\text { Triangular grid }\end{array}$ & $\begin{array}{c}\text { Our results } \\
\text { Rectangular grid }\end{array}$ \\
\hline$\widehat{\mathrm{Nu}}_{0}$ & $0.0012 \%$ & $0.056 \%$ & $0.0012 \%$ & $0.099 \%$ & $0.025 \%$ & $0.027 \%$ & 8.6867 \\
\hline$\widehat{\mathrm{Nu}}_{1}$ & $0.0012 \%$ & $0.014 \%$ & $0.14 \%$ & $0.61 \%$ & $0.007 \%$ & $0.027 \%$ & 8.6867 \\
\hline $\mathrm{Nu}(0.0 .5)$ & $0.0013 \%$ & $0.054 \%$ & $0.016 \%$ & $0.055 \%$ & N.A & $0.05 \%$ & 7.4592 \\
\hline $\mathrm{Nu}(1.0 .5)$ & $0.002 \%$ & $0.25 \%$ & $0.024 \%$ & $0.55 \%$ & N.A & $0.07 \%$ & 8.6370 \\
\hline $\max _{y}(\mathrm{Nu}(0 . y))$ & $0.016 \%$ & $0.18 \%$ & $0.032 \%$ & $0.24 \%$ & $0.15 \%$ & $0.01 \%$ & 20.2737 \\
\hline $\min _{y}(\mathrm{Nu}(0 ; y))$ & $0.028 \%$ & $0.64 \%$ & $0 \%$ & $0.019 \%$ & $0.037 \%$ & $0.15 \%$ & 1.0670 \\
\hline $\max _{y}(\mathrm{Nu}(1 ; y))$ & $0.014 \%$ & $0.34 \%$ & $0.25 \%$ & $0.91 \%$ & $0.093 \%$ & $0.45 \%$ & 15.5216 \\
\hline $\min _{y}(\mathrm{Nu}(1 ; y))$ & $0.040 \%$ & $0.34 \%$ & $0.052 \%$ & $0.26 \%$ & $0.15 \%$ & $1 \%$ & 0.7578 \\
\hline$P$ & $0.00075 \%$ & $0.11 \%$ & $0.0019 \%$ & $0.034 \%$ & $0.045 \%$ & $0.02 \%$ & 0.924482 \\
\hline Structured grid & $2048 \times 2048$ & $320 \times 320$ & $169 \times 148$ & $160 \times 160$ & & & $304 \times 304$ \\
\hline Number of cells & $4.2 \times 10^{6}$ & 102,400 & 25,012 & 25,600 & 200,000 & 173,030 & 92,416 \\
\hline
\end{tabular}




\begin{tabular}{|c|c|c|c|c|c|}
\hline & Vierendeels & Dabbene & Kloczko & Heuveline & Our results \\
\hline$\widehat{\mathrm{Nu}}_{0}$ & $0 . \%$ & $0.086 \%$ & $0.38 \%$ & $0.006 \%$ & 16.2409 \\
\hline$\widehat{\mathrm{Nu}}_{1}$ & $0 . \%$ & $0.32 \%$ & $0.050 \%$ & $0.10 \%$ & 16.2409 \\
\hline $\mathrm{Nu}(0,0.5)$ & $0 . \%$ & $2.6 \%$ & $0.068 \%$ & N.A & 13.1886 \\
\hline $\mathrm{Nu}(1,0.5)$ & $0.0064 \%$ & $1.6 \%$ & $0.87 \%$ & N.A & 15.5113 \\
\hline $\max _{y}(\mathrm{Nu}(0, y))$ & $0.03 \%$ & $0.31 \%$ & $1.3 \%$ & $0.15 \%$ & 46.3941 \\
\hline $\min _{y}(\mathrm{Nu}(0, y))$ & $0.068 \%$ & $0.068 \%$ & $1 \%$ & $0 . \%$ & 1.45475 \\
\hline $\max _{y}(\mathrm{Nu}(1, y))$ & $0.032 \%$ & $1 . \%$ & $0.38 \%$ & $0.35 \%$ & 34.2832 \\
\hline $\min _{y}(\mathrm{Nu}(1, y))$ & $0 . \%$ & $0.09 \%$ & $2.8 \%$ & $0.09 \%$ & 1.08878 \\
\hline$P$ & $0.003 \%$ & $0.04 \%$ & $0.04 \%$ & $0.04 \%$ & 0.92261 \\
\hline Structured grid & $2048 \times 2048$ & $160 \times 160$ & $100 \times 100$ & & $304 \times 304$ \\
\hline Number of cells & $4.2 \times 10^{6}$ & 25,600 & 10,000 & 400,000 & 92,416 \\
\hline
\end{tabular}

Table 2

Comparison with results given in [19] for $R a=10^{7}$ 